\title{
Monkey's Revenge: Coordinate Geometry Learning Environment with Game-Like Elements
}

\author{
Dovan Rai and Joseph E. Beck \\ Computer Science Department, Worcester Polytechnic Institute \\ \{dovan, josephbeck\} @wpi .edu
}

Educational games intend to make learning more enjoyable, but at the potential cost of compromising learning efficiency. Therefore, instead of creating educational games, we have created a learning environment with game-like elements: the elements of games that are engaging. Our approach is to assess each game-like element in terms of benefits such as enhancing engagement as well as its costs such as sensory or working memory overload, with the goal of maximizing both engagement and learning. We created Monkey's Revenge, a coordinate geometry learning environment with game -like elements such as narrative, immediate visual feedback, personalization, collecting badges, etc. The tutor basically consists of a series of 8th grade (approximately 13-year olds) coordinate geometry problems wrapped in a visual cover story. In the narrative, Mike, a boy is thrown out of class for playing a game on his cell phone and encounters a monkey and they become friends. He builds a house for the monkey, but the monkey is not eager to become domesticated and destroys the house, steals his phone and runs away. The boy tries to get back his phone by throwing balls to the monkey. To move the story forward, the students have to solve coordinate problems like calculating distance between the boy and the monkey, slope of the roof and walls of the house, finding points where the monkey tied to a rope cannot find bananas and finally figure out slopes, intercepts and equation of the line of the path of the ball. The math content gets more advanced as a student progresses through the story. Students get immediate visual feedback on their response. For example, if a student puts banana at the wrong coordinate, the monkey can reach it and will eat the banana. We are using a very simple and minimalistic approach so as not to overwhelm students who are already struggling with the content.

We built four versions of this tutor with different degree of "game-like" (one without visual feedback, one without narrative and a basic tutor with the same hints and bug messages as the other three versions). Based on a study with 297 students, that students who had more "game-like" tutor reported more liking of the tutor but we found no conclusive difference in learning gain. We had made a very conservative progression from tutor towards game adding as little detail as possible. So, our first concern was to attain optimal engagement so as not to leave students disenchanted. Based on our next study focusing on learning gain, we will decide whether we have to enhance or scale back game-like elements. With such iterative process, we aim to find a "sweet spot" in the tutor game space where we can find optimal engagement and learning. 